\title{
PROPRIEDADES DE PAINÉIS AGLOMERADOS FABRICADOS COM PARTÍCULAS DE EUCALIPTO (Eucalyptus urophylla), PARICÁ (Schizolobium amazonicum) E VASSOURA (Sida spp.)
}

\author{
Juliana Jerásio Bianche ${ }^{1}$, Angélica de Cássia Oliveira Carneiro², Benedito Rocha Vital ${ }^{3}$, Flávia Alves Pereira ${ }^{1}$, \\ Rosimeire Cavalcante dos Santos ${ }^{4}$, Déborah Nava Soratto ${ }^{5}$
}

(recebido: 1 de setembro de 2010; aceito: 29 de junho de 2012)

\begin{abstract}
RESUMO: O gênero Sida pode se tornar uma fonte alternativa bastante promissora para a produção de painéis de madeira aglomerada, uma vez que é utilizado na fabricação de cordas, em razão da qualidade de suas fibras, porém, são inexistentes pesquisas relacionadas à produção de painéis de aglomerado, utilizando este material lignocelulósico. Neste trabalho, objetivou-se avaliar as propriedades físicas e mecânicas de painéis aglomerados de eucalipto (Eucalyptus urophylla) e paricá (Schizolobium amazonicum) em associação com vassoura (Sida spp.). Para a produção dos painéis de aglomerado foram utilizadas misturas em 4 proporções $(25,50,75$ e $100 \%)$ de partículas de vassoura em associação com partículas de eucalipto e paricá, e associação de partículas de eucalipto em associação com paricá, sendo adicionadas a essas partículas dois teores de adesivo à base de uréiaformaldeído (6\% e 8\%). Os ensaios físicos e mecânicos foram realizados segundo a norma NBR/ABNT 14810-3 (ASSOCIAÇÃO BRASILEIRA DE NORMAS TÉCNICAS - ABNT, 2002). Os resultados experimentais mostraram que o aumento no teor de adesivo na produção dos painéis de aglomerado contribuiu para melhorar algumas propriedades físicas e mecânicas. Concluiu-se que o incremento de partículas de vassoura nos painéis teve efeito diferenciado em função das espécies utilizadas. O incremento da porcentagem de partículas de vassoura aos painéis produzidos com eucalipto não afetou a resistência à tração perpendicular, arrancamento de parafuso e dureza Janka. $\mathrm{O}$ incremento da porcentagem de partículas de vassoura aos painéis produzidos com paricá não afetou a resistência à dureza Janka e ao arrancamento de parafuso. Os painéis produzidos com partículas de vassoura absorveram mais água e, por consequência, o inchamento em espessura aumentou, sendo, portanto não recomendados para uso em ambientes com alto teor de umidade. Concluiu-se que o gênero Sida, de modo geral, apresentou potencial para produção dos painéis de aglomerado.
\end{abstract}

Palavras-chave: Painel, madeira, uréia-formaldeído.

\section{PROPERTIES OF PARTICLEBOARDS FABRICATED WITH EUCALYPTUS (Eucalyptus urophylla), PARICA (Schizolobium amazonicum) AND VASSOURA (Sida spp.) PARTICLES}

\begin{abstract}
The genus Sida may become a promising alternative source for the production of particleboards. Nowadays it is used for manufacturing ropes due to the quality of its fibers, however, there is no research relating the production of particleboards using this lignocelluloses material. This study aimed to evaluate the effect of eucalyptus (Eucalyptus urophylla), paricá (Schizolobium amazonicum) and vassoura (Sida spp.) on the physical and mechanical properties of particleboard. Particleboard contained 25, 50, 75 and $100 \%$ of eucalyptus and paricá or eucalytus and vassoura or paricá an vassoura were fabricated. Two (6\% and 8\%) amounts of urea-formaldehyde adhesive were used. The physical and mechanical properties were determined according to NBR/ABNT 148103 (ASSOCIAÇÃO BRASILEIRA DE NORMAS TÉCNICAS - ABNT, 2002). It was observed that increase in resin content in the improved some physical and mechanical properties. It was concluded that the amount of vassoura particles on panels had different effects depending on the species used. The increase in the percentage of vassoura particles in the Eucalyptus panels did not affect the internal bond, screw withdrawal and hardness. Increasing the percentage of vassoura particles on the panels produced with paricá did not affect its hardness and resistance to screw withdrawal. The panels produced with vassoura particle absorbed more water and, consequently, increased the thickness swelling, and therefore is not recommended for use in environments with high humidity. It was concluded that the genus Sida, in general, has potential for particleboard production.
\end{abstract}

Key words: Panel, wood, urea-formaldehyde.

'Engenheira Florestal, Doutoranda em Ciência Florestal - Universidade Federal de Viçosa/UFV - Departamento de Engenharia Florestal Campus Universitário - 36570-000 - Viçosa, MG, Brasil - jubianche@yahoo.com.br, flaviafloresta@yahoo.com.br

${ }^{2}$ Engenheira Florestal, Professora DSc. em Ciência Florestal - Universidade Federal de Viçosa/UFV - Departamento de Engenharia Florestal - Campus Universitário - 36570-000 - Viçosa, MG, Brasil - cassiacarneiro@ufv.br

${ }^{3}$ Engenheiro Florestal, Professor DSc. em Produtos Florestais - Universidade Federal de Viçosa/UFV - Departamento de Engenharia Florestal - Campus Universitário - 36570-000 - Viçosa, MG, Brasil - bvital@ufv.br

${ }^{4}$ Engenheira Florestal, Professora DSc. em Ciência e Tecnologia da Madeira - Universidade Federal do Rio Grande do Norte/UFRN Escola Agrícola de Jundiaí - RN 160, Km 03, Distrito de Jundiaí - Cx. P. 07 - 59280-000 - Macaíba, RN - meire_caico@yahoo.com.br ${ }^{5}$ Engenheira Florestal, Mestre em Ciência Florestal - Universidade Federal de Viçosa/UFV - Departamento de Engenharia Florestal Campus Universitário - 36570-000 - Viçosa, MG, Brasil - deborahsoratto@msn.com 


\section{INTRODUÇÃO}

A mistura de espécies é uma prática possível de ser empregada com êxito, permitindo que espécies de massas específicas mais elevadas sejam combinadas com espécies de massas específicas mais baixas, produzindo painéis resistentes. A utilização total ou parcial de novas espécies na composição dos painéis se torna uma alternativa rápida, de baixo custo e sustentável. Dentre essas espécies, podem ser citadas o paricá (Schizolobium amazonicum) e a vassoura (Sida spp.) (BIANCHE, 2009). A madeira de paricá vem sendo utilizada no Norte do Brasil, principalmente, para a fabricação de compensados, apresentando potencial para a produção de painéis de aglomerado. Coli (2007), estudando as propriedades físicas e mecânicas dos painéis de partículas com madeira de paricá e fibra de coco, concluiu que a madeira de paricá é apropriada para a confecção de painéis de aglomerado. Naumann et al. (2008), avaliando as propriedades de painéis de madeira aglomerada, fabricados com partículas de Eucalyptus urophylla e Schizolobium amazonicum, verificaram que a utilização de um porcentual maior de partículas de paricá, aliada ao aumento da taxa de compactação, melhorou a estabilidade dimensional dos painéis e resultou em maior resistência mecânica. $\mathrm{Na}$ pesquisa desenvolvida com a mistura de madeiras de Pinus elliottii e Mimosa scabrella, Iwakiri et al. (1995) concluíram que a mistura dessas espécies, em proporção de $50 \%$ cada, resulta em painéis com melhores propriedades físicomecânicas. Os autores ressaltam a importância desses resultados, tendo em vista a viabilidade de melhor aproveitamento da madeira de Mimosa scabrella, utilizada principalmente como lenha e carvão, em produto de maior valor agregado. Santos (2008), estudando a produção de aglomerado convencional a partir de partículas da madeira de candeia, (após a extração do óleo), em associação com madeiras de eucalipto e pinus, concluiu que o resíduo da madeira de candeia se mostrou viável tecnicamente na confecção de painéis de aglomerado convencional em todas as propriedades, exceto para valores de MOE e MOR.

Em razão das possíveis variações nos valores da densidade da madeira entre as espécies, variáveis como a razão de compactação, a área superficial das partículas e a disponibilidade de resina por unidade de área das partículas estarão envolvidas na análise comportamental dos painéis (IWAKIRI et al., 1990). Em relação à mistura de espécies, Vital (1973) concluiu que a mistura de madeiras de diferentes densidades se apresenta como alternativa para o aproveitamento de madeiras com maiores densidades, resultando em painéis com densidade e propriedades aceitáveis pelo mercado. Essa mistura de espécies pode representar uma saída para o aproveitamento de madeiras tropicais, de maiores e menores densidades.

O gênero Sida, pertencente à família das Malváceas, abriga muitas espécies vulgarmente conhecidas por vassouras, vassourinhas, guaxumas ou guanxumas, malvabrava, chá-da-índia (LORENZI, 2000). Algumas espécies são utilizadas na fitoterapia popular ou substituem a juta na produção de cordas e sacos de aniagem, em razão da resistência de suas fibras (CALDERAN, 2007). No Brasil, está distribuída nas regiões Sul, Sudeste e Centro-Oeste, sendo considerada uma importante erva daninha na agricultura, principalmente em pastagens, pois o fato de não ser palatável ao gado favorece sua permanência e multiplicação nos campos (BACCHI et al., 1982; LORENZI, 2000). O gênero Sida pode se tornar uma fonte alternativa promissora para a produção de painéis, uma vez que é utilizado na fabricação de cordas, pela resistência de suas fibras. Portanto, a utilização dessa planta para a produção de painéis aglomerados poderá contribuir para melhorar as propriedades físicas e mecânicas. Entretanto, não há dados relacionados a capacidade produtiva da vassoura, mas o fato dessa planta ser daninha, contribui para a sua multiplicação e permanência nos campos, o que permite a sua utilização como matéria-prima para produção de painel aglomerado. Porém, são inexistentes pesquisas referentes ao material lignocelulósico desse gênero para a produção de painéis, fazendo-se necessário, então, estudos para a qualificação desta matéria-prima e posterior produção dos painéis, sendo esse gênero utilizado em associação com outros materiais, ou isoladamente.

Diante do exposto, o objetivo geral do presente trabalho foi avaliar as propriedades físicas e mecânicas de aglomerado fabricado com partículas de eucalipto (Eucalyptus urophylla), e paricá (Schizolobium amazonicum) em associação com vassoura (Sida spp.). Os objetivos específicos foram:

a) Determinar a influência do uso de material lignocelulósico de vassoura em associação com madeiras de eucalipto e paricá nas propriedades dos painéis.

b) Avaliar o efeito do teor de adesivo de uréiaformaldeído sobre os painéis de aglomerado.

\section{MATERIAL E MÉTODOS}

O experimento foi realizado nos Laboratórios de Painéis e Energia da Madeira, e Propriedades da Madeira,

Cerne, Lavras, v. 18, n. 4, p. 623-630, out./dez. 2012 
da Universidade Federal de Viçosa, em Viçosa, Estado de Minas Gerais. A madeira de paricá foi oriunda de plantios comerciais da região de Dom Eliseu, no Estado do Pará. A madeira de Eucalyptus urophylla foi obtida de plantios comerciais da empresa Arcelor Mittal, situado no Estado de Minas Gerais. O material lenhoso de Sida spp. foi coletado com o caule, folha e raiz, nas áreas da Silvicultura e Dendrologia, do Departamento de Engenharia Florestal, da Universidade Federal de Viçosa, em Viçosa, Minas Gerais. Para a produção das partículas de vassoura, foram moídos em moinho de martelo, sem utilização de peneira, o caule e a raiz. Para a produção das partículas de eucalipto e paricá, foram utilizadas tábuas e costaneiras, as quais foram desdobradas em baguetas com $2 \mathrm{~cm}$ de espessura e $9 \mathrm{~cm}$ de largura. Posteriormente, as baguetas foram submersas em água por sete dias. Após a saturação em água, as baguetas foram processadas em um moinho de facas, produzindo-se flocos. Foi utilizado um moinho de martelo para processar os flocos e gerar as partículas. Para determinar a densidade básica da vassoura foram utilizadas amostras do caule com $5 \mathrm{~cm}$ de comprimento, uma vez que não teria como selecionar amostras utilizando a largura. Para as madeiras de eucalipto e paricá foram utilizadas amostras com $5 \mathrm{~cm}$ de comprimento e $5 \mathrm{~cm}$ de largura. As densidades básicas da vassoura, eucalipto e paricá foram determinadas utilizando o método de imersão em água, segundo metodologia proposta por Vital (1984). Os painéis foram produzidos utilizando-se três matérias-primas (eucalipto, paricá e vassoura) em quatro proporções $(25,50,75$ e $100 \%)$ de partículas de vassoura em associação com partículas de eucalipto e paricá, e partículas de eucalipto em associação com paricá. Foram utilizados dois teores de adesivo à base de uréia-formaldeído $(6 \%$ e $8 \%$ ), base peso seco das partículas, com três repetições, resultando em 24 tratamentos e 72 painéis de aglomerado. Não foi adicionada parafina às partículas para não influenciar nos resultados dos testes físicos e mecânicos dos painéis, uma vez que não se conhecia o comportamento da partícula de vassoura. Das partículas de paricá, eucalipto e vassoura, foram produzidos painéis de aglomerado com dimensões de $40 \times 40 \times 1,00 \mathrm{~cm}$, com densidade nominal igual a $0,65 \mathrm{~g} / \mathrm{cm}^{3}$. De acordo com o tratamento, as partículas de paricá, eucalipto e vassoura foram distribuídas para a formação do colchão, tentando-se obter um colchão mais uniforme possível para evitar diferenças de gradiente no painel. Isso foi difícil de obter, já que ocorreu o enovelamento das partículas de vassoura (Figura 1), gastando muito tempo para formação do colchão, por isso não foi adicionado catalisador ao adesivo, pois este iria curar antes de ir para a prensa. Todos os painéis foram produzidos numa prensa de laboratório, marca INCO, empregando-se uma pressão de $32 \mathrm{kgf} / \mathrm{cm}^{2}$, a uma temperatura de $170^{\circ} \mathrm{C}$, por 8 minutos, contados a partir do fechamento da prensa.

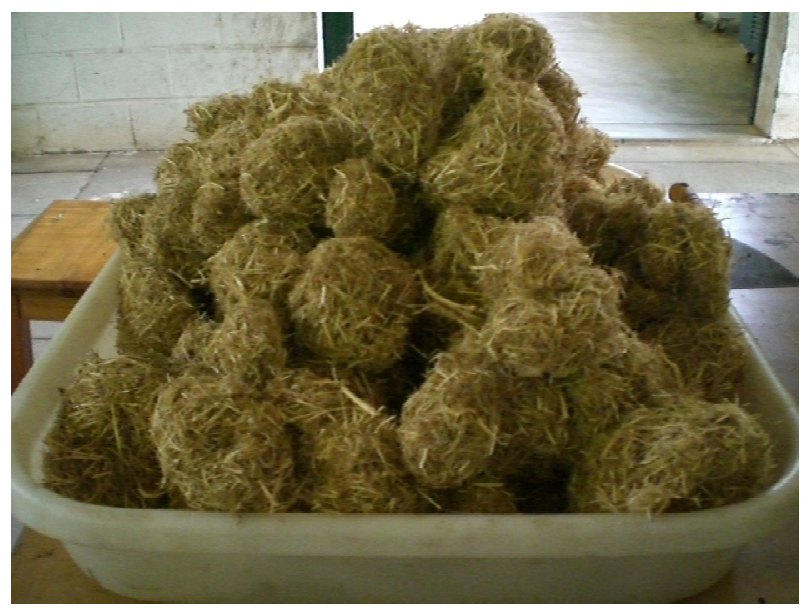

Figura 1 - Enovelamento das partículas de vassoura.

Figure 1 - Vassoura particles balls.

Foram confeccionados os corpos-de-prova para os testes físicos e mecânicos, que foram climatizados até o equilíbrio, a uma umidade relativa de $65 \pm 5 \%$ e a uma temperatura de $203^{\circ} \mathrm{C}$, conforme estabelecido pela norma ABNT NBR 14810-3 (ABNT, 2002). Determinaram-se as propriedades físicas e mecânicas dos painéis, conforme a norma da ABNT: NBR 14810-3 (ABNT, 2002), e os valores médios de resistência mecânica foram comparados com os valores mínimos exigidos pela norma comercial ANSI/A1208.1-1993 (AMERICAN NATIONALSTANDARD - ANS, 1993), e os dos testes físicos, comparados com os valores exigidos pela norma DIN 68761 (1) (SANTANA; PASTORE, 1981), para absorção e inchamento. Os resultados das propriedades dos painéis foram submetidos à análise de variância (ANOVA), quando estabelecidas diferenças significativas, os tratamentos foram comparados entre si por meio do teste de Tukey, a 5\% de probabilidade. Utilizouse o software SAEG.

\section{RESULTADOS E DISCUSSÃO}

\subsection{Densidade básica das matérias-primas}

Os valores médios de densidade básica das matériasprimas: paricá, vassoura e eucalipto, utilizadas na confecção dos painéis, estão apresentados na Tabela 1 .

Cerne, Lavras, v. 18, n. 4, p. 623-630, out./dez. 2012 
Tabela 1 - Valores médios da densidade básica do paricá (Schizolobium amazonicum) vassoura (Sida spp.) e eucalipto (Eucalyptus urophylla).

Table 1 - Paricá (Schizolobium amazonicum), vassoura (Sida spp.) and eucalyptus (Eucalyptus urophylla) densitymean values.

\begin{tabular}{lc}
\hline Matérias-primas & Densidade básica média $\left(\mathrm{g} / \mathrm{cm}^{3}\right)$ \\
\hline Schizolobium amazonicum & $0.26 \mathrm{~B}$ \\
Sida spp. & $0.46 \mathrm{~A}$ \\
Eucalyptus urophylla & $0.56 \mathrm{~A}$ \\
\hline
\end{tabular}

Médias seguidas da mesma letra na coluna não diferem entre si pelo teste de Tukey a 5\% de significância.
Observa-se, na Tabela 1, que não houve diferença significativa entre as densidades da vassoura e do eucalipto, somente a densidade do paricá diferiu estatisticamente das demais.

\subsection{Propriedades dos painéis}

\subsubsection{Propriedades físicas}

Os resultados das propriedades físicas dos painéis de aglomerado e destas em função do teor de adesivo, estão apresentados nas Tabelas 2 e 3, respectivamente.

Tabela 2 - Valores médios das propriedades físicas dos painéis de aglomerado, em função da composição das partículas.

Table 2 - Particleboards mean physical properties as function of particles composition.

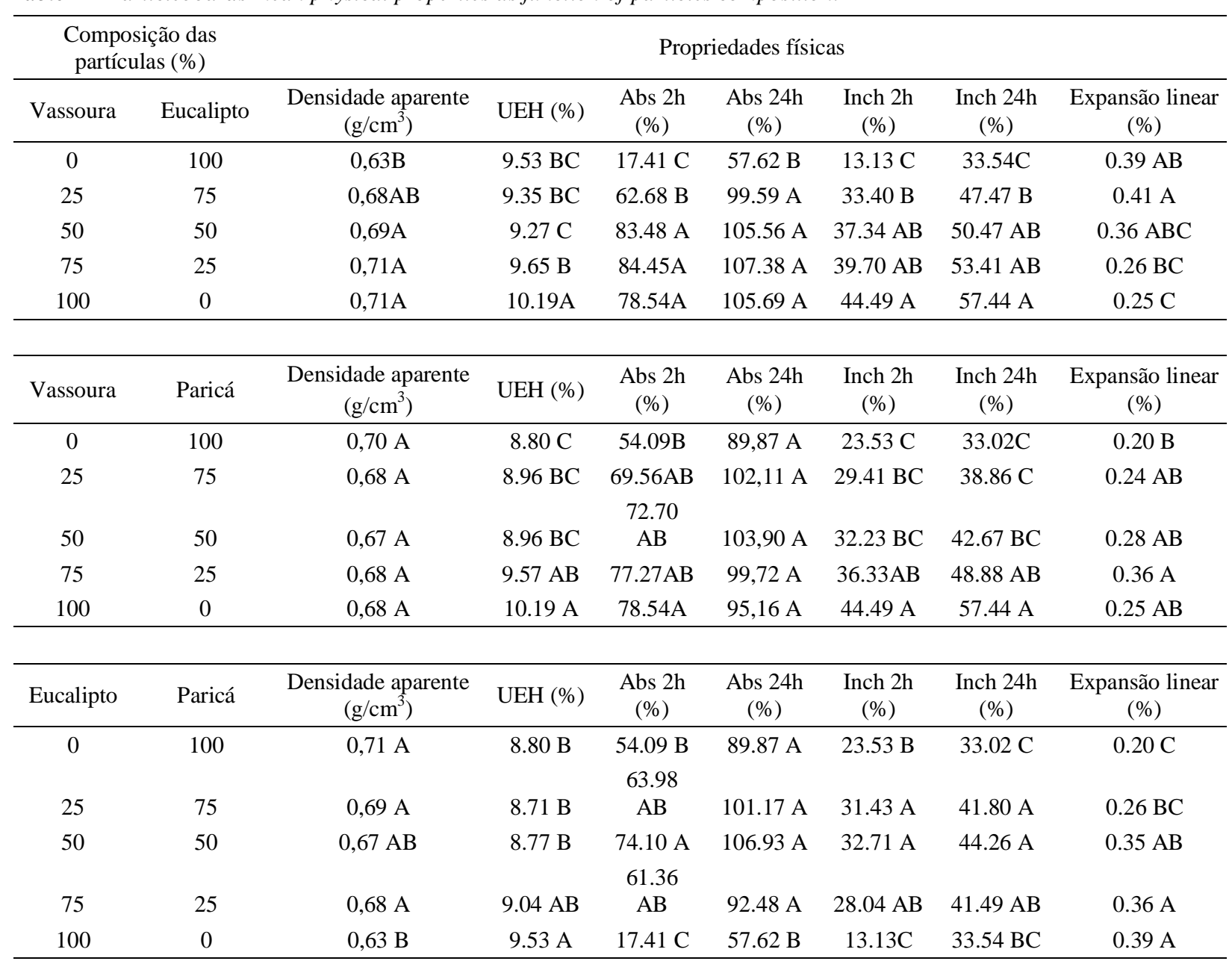

Médias seguidas pela mesma letra na coluna para cada composição não diferem entre si pelo teste Tukey a $5 \%$ de significância. $\mathrm{UEH}=$ Umidade de equílibrio higroscópico. Abs= Absorção de água. Inch= Inchamento em espessura.

\section{Cerne, Lavras, v. 18, n. 4, p. 623-630, out./dez. 2012}


Tabela 3 - Valores médios (desvio padrão) das propriedades físicas dos painéis de aglomerado em função do teor de adesivo.

Table 3 - Mean values and standard deviation of the particleboards physical properties as affected by adhesive resin content.

\begin{tabular}{lcc}
\hline \multirow{2}{*}{ Propriedades físicas } & \multicolumn{2}{c}{ Teor de adesivo } \\
\cline { 2 - 3 } & $6 \%$ & $0 \%$ \\
\hline Densidade aparente & $0,68 \mathrm{~A}(0,035)$ & $9,29 \mathrm{~A}(0,035)$ \\
Umidade de equilíbrio higroscópico & $9,17 \mathrm{~A}(0,47)$ & $59,24 \mathrm{~B}(20,61)$ \\
Absorção 2h & $74,03 \mathrm{~A}(19,55)$ & $91,52 \mathrm{~B}(17,70)$ \\
Absorção 24h & $104,68 \mathrm{~A}(15,70)$ & $27,50 \mathrm{~B}(8,28)$ \\
Inchamento 2h & $36,12 \mathrm{~A}(9,72)$ & $39,12 \mathrm{~B}(7,57)$ \\
Inchamento 24h & $49,77 \mathrm{~A}(9,76)$ & $0,29 \mathrm{~B}(0,09)$ \\
Expansão linear & $0,33 \mathrm{~A}(0,09)$ &
\end{tabular}

Médias seguidas pela mesma letra na linha não diferem entre si pelo teste Tukey a $5 \%$ de significância.

Para a produção dos painéis foi utilizada uma mesma massa de partículas, para obter uma densidade nominal igual a $0,65 \mathrm{~g} / \mathrm{cm}^{3}$, entretanto as diferenças entre a geometria das partículas de eucalipto, paricá e vassoura, fez com que fosse obtido um volume de partículas diferente, sendo aplicada a este maior ou menor taxa de compactação, obtendo, dessa forma, diferenças entre as densidades aparentes dos painéis. Como observado na Tabela 2, todos os painéis apresentaram valores médios de densidade aparente acima do valor estabelecido para a densidade nominal no plano experimental, exceto os produzidos apenas com partículas de eucalipto, que obtiveram densidade inferior. Isso se deve ao fato da maior densidade da madeira de eucalipto em relação à madeira de paricá e ao caule de vassoura, gerando um volume menor de partículas para a formação do colchão, necessitando de menor taxa de compactação na prensa, fornecendo um painel com menor densidade. Apesar de não ter havido diferenças significativas, foi verificado que, aumentando a quantidade de vassoura na composição do painel (vassoura $\mathrm{x}$ eucalipto), ocorreu um aumento na sua densidade, isso se deve à menor densidade das partículas de vassoura, que requerem aumento na taxa de compactação. Segundo a norma ANSI/A 208.1/93 (ANS, 1993), os painéis obtidos neste trabalho são classificados como painéis de média densidade, que correspondem à faixa de densidade de $0,64 \mathrm{~g} / \mathrm{cm}^{3}$ a 0,80 $\mathrm{g} / \mathrm{cm}^{3}$. Observou-se que a quantidade de adesivo utilizada na produção dos painéis não influenciou sua densidade (Tabela 3), evidenciando que os fatores que mais influenciam na densidade do painel são a densidade da madeira e, consequentemente, a taxa de compactação utilizada no processo de prensagem. Os painéis absorveram mais água que o prescrito pela norma DIN 68m 761 (1) (SANTANA; PASTORE, 1981), cujo valor deve estar abaixo de $10 \%$ e $15 \%$ para duas e vinte quatro horas, respectivamente. Deve-se salientar que os painéis produzidos neste trabalho não receberam parafina, o que, provavelmente, diminuiria os valores médios de absorção. Os valores médios de expansão linear ficaram abaixo do máximo permitido pela norma ANSI/A 208.1/93 (ANS, 1993), que estabelece valores abaixo de $0,35 \%$. Para as composições vassoura $\mathrm{x}$ eucalipto e vassoura x paricá, com o aumento da porcentagem de vassoura observouse um aumento no teor de umidade dos painéis, mostrando que a partícula de vassoura é mais higroscópica. Esse fato pode ser atribuído à maior área superficial da partícula de vassoura em relação às partículas de paricá e eucalipto, o que favorece maior absorção de água. De acordo com a Tabela 2, observa-se que não houve efeito significativo das partículas de vassoura na absorção de água após duas e vinte quatro horas de imersão. Somente os painéis produzidos apenas com partículas de eucalipto obtiveram, de forma significativa, menores absorções. Fato atribuído pela melhor formação do colchão para os painéis de eucalipto, diminuindo os espaços vazios entre as partículas, permitindo maior ligação entre elas, reduzindo a absorção de água.

\subsection{Propriedades mecânicas dos painéis}

Os valores médios para as propriedades mecânicas dos painéis e destas em função do teor de adesivo, estão apresentados nas Tabelas 4 e 5, respectivamente.

Cerne, Lavras, v. 18, n. 4, p. 623-630, out./dez. 2012 
Tabela 4 - Valores médios das propriedades mecânicas dos painéis em função da composição das partículas.

Table 4 - Particleboard mechanical properties mean values according to particles composition.

\begin{tabular}{|c|c|c|c|c|c|c|}
\hline \multicolumn{2}{|c|}{ Composição das partículas (\%) } & \multicolumn{5}{|c|}{ Propriedades Mecânicas } \\
\hline Vassoura & Eucalipto & $\begin{array}{l}\text { Tração } \\
\text { (MPA) }\end{array}$ & $\begin{array}{l}\text { MOR } \\
(\mathrm{MPa})\end{array}$ & $\begin{array}{l}\mathrm{MOE} \\
(\mathrm{MPa})\end{array}$ & $\begin{array}{l}\text { Dureza } \\
(\mathrm{MPa})\end{array}$ & $\begin{array}{c}\text { Arrancamento } \\
\text { (N) }\end{array}$ \\
\hline 0 & 100 & $0,37 \mathrm{~A}$ & $10,88 \mathrm{~B}$ & $1383,81 \mathrm{AB}$ & $34,07 \mathrm{~B}$ & $1047,45 \mathrm{~A}$ \\
\hline 25 & 75 & $0,47 \mathrm{~A}$ & $14,05 \mathrm{AB}$ & $1605,20 \mathrm{~A}$ & $41,63 \mathrm{AB}$ & $1076,20 \mathrm{~A}$ \\
\hline 50 & 50 & $0,49 \mathrm{~A}$ & $15,52 \mathrm{~A}$ & $1548,27 \mathrm{~A}$ & $43,01 \mathrm{AB}$ & $1122,21 \mathrm{~A}$ \\
\hline 75 & 25 & $0,51 \mathrm{~A}$ & $15,54 \mathrm{~A}$ & $1520,83 \mathrm{AB}$ & $43,76 \mathrm{~A}$ & $1122,59 \mathrm{~A}$ \\
\hline 100 & 0 & $0,49 \mathrm{~A}$ & $13,13 \mathrm{AB}$ & $1154,15 \mathrm{~B}$ & $39,97 \mathrm{AB}$ & $1137,74 \mathrm{~A}$ \\
\hline Vassoura & Paricá & $\begin{array}{l}\text { Tração } \\
(\mathrm{MPa})\end{array}$ & $\begin{array}{l}\text { MOR } \\
(\mathrm{MPa})\end{array}$ & $\begin{array}{l}\mathrm{MOE} \\
(\mathrm{MPa})\end{array}$ & $\begin{array}{c}\text { Dureza } \\
(\mathrm{MPa})\end{array}$ & $\begin{array}{c}\text { Arrancamento } \\
\text { (N) }\end{array}$ \\
\hline 0 & 100 & $0,75 \mathrm{~A}$ & $26,44 \mathrm{~A}$ & $2955,69 \mathrm{~A}$ & $50,52 \mathrm{~A}$ & $1376,00 \mathrm{~A}$ \\
\hline 25 & 75 & $0,46 \mathrm{~B}$ & $22,22 \mathrm{AB}$ & $2344,72 \mathrm{AB}$ & $44,34 \mathrm{~A}$ & $1153,90 \mathrm{~A}$ \\
\hline 50 & 50 & $0,47 \mathrm{~B}$ & $20,25 \mathrm{AB}$ & $2118,58 \mathrm{~B}$ & $40,60 \mathrm{~A}$ & $1131,49 \mathrm{~A}$ \\
\hline 75 & 25 & $0,43 \mathrm{~B}$ & $18,58 \mathrm{BC}$ & $1607,17 \mathrm{BC}$ & $41,51 \mathrm{~A}$ & $1136,92 \mathrm{~A}$ \\
\hline 100 & 0 & $0,34 \mathrm{~B}$ & $13.13 \mathrm{C}$ & $1154,16 \mathrm{C}$ & $40,49 \mathrm{~A}$ & $1027,51 \mathrm{~A}$ \\
\hline Eucalipto & Paricá & $\begin{array}{l}\text { Tração } \\
(\mathrm{MPa})\end{array}$ & $\begin{array}{l}\text { MOR } \\
(\mathrm{MPa})\end{array}$ & $\begin{array}{l}\mathrm{MOE} \\
(\mathrm{MPa})\end{array}$ & $\begin{array}{c}\text { Dureza } \\
(\mathrm{MPa})\end{array}$ & $\begin{array}{c}\text { Arrancamento } \\
\text { (N) }\end{array}$ \\
\hline 0 & 100 & $0,75 \mathrm{~A}$ & $26,44 \mathrm{~A}$ & $2955,69 \mathrm{~A}$ & $50,52 \mathrm{~A}$ & $1376,00 \mathrm{~A}$ \\
\hline 25 & 75 & $0,65 \mathrm{~A}$ & 20,73 B & $2335,05 \mathrm{~B}$ & $44,26 \mathrm{AB}$ & $1162,36 \mathrm{AB}$ \\
\hline 50 & 50 & $0,57 \mathrm{AB}$ & $17,34 \mathrm{BC}$ & $1977,85 \mathrm{BC}$ & $39,81 \mathrm{BC}$ & $1153,38 \mathrm{AB}$ \\
\hline 75 & 25 & $0,65 \mathrm{~A}$ & $15,77 \mathrm{C}$ & $1799,16 \mathrm{C}$ & $43,32 \mathrm{AB}$ & $1175,10 \mathrm{AB}$ \\
\hline 100 & 0 & $0,38 \mathrm{~B}$ & $10,88 \mathrm{D}$ & $1383,81 \mathrm{D}$ & $34,07 \mathrm{C}$ & $1047,46 \mathrm{~B}$ \\
\hline
\end{tabular}

Médias seguidas pela mesma letra na coluna, para uma mesma composição, não diferem entre si pelo teste Tukey a 5\% de significância.

MOR $=$ Módulo de ruptura. MOE $=$ Módulo de elasticidade.

Tabela 5 - Valores médios (desvio padrão) das propriedades mecânicas dos painéis em função do teor de adesivo.

Table 5 - Particleboard mechanical properties mean values and standard deviation as affected by resin content.

\begin{tabular}{lcc}
\hline \multirow{2}{*}{ Propriedades mecânicas } & \multicolumn{3}{c}{ Teor de adesivo } \\
\cline { 2 - 3 } & $6 \%$ & $8 \%$ \\
\hline Tração perpendicular (MPa) & $0,41 \mathrm{~B}(0,15)$ & 0,55 A $(0,20)$ \\
Módulo de ruptura (MPa) & 17,03 A $(4,98)$ & 18,05 A $(5,47)$ \\
Módulo de elasticidade (MPa) & 1795,64 B $(534,56)$ & 1929,44 A $(626,90)$ \\
Dureza Janka (MPa) & 40,81 A $(5,88)$ & 43,51 A $(7,51)$ \\
Arrancamento (N) & 1092,70 A $(186,52)$ & 1133,67 A $(213,77)$ \\
\hline
\end{tabular}

Médias seguidas pela mesma letra na linha não diferem entre si pelo teste Tukey a 5\% de significância.

\section{Cerne, Lavras, v. 18, n. 4, p. 623-630, out./dez. 2012}


Verificou-se que, aumentando a porcentagem de partículas de vassoura na composição, ocorreu uma queda na resistência à tração dos painéis. Isso pode ser atribuído à etapa de formação do colchão, em razão do enovelamento das partículas de vassoura, dificultando a homogeneidade do colchão, gerando espaços vazios. De acordo com Vital et al. (1992), as partículas mais espessas melhoram as propriedades de tração perpendicular, enquanto, segundo Hse (1988 citado por KELLY, 1977), partículas maiores respondem melhor à alta compactação. Isso ocorre porque, para uma mesma massa de partículas, aquelas mais espessas e curtas, no caso, as partículas de paricá, possuem menor área superficial e recebem maior quantidade de adesivo por unidade de área, apresentando maior ligação entre as partículas, resultando em maior valor de ligação interna. Os valores médios apresentados na Tabela 5 mostraram que os painéis apresentaram valores acima da norma ANSI/A 208.1/93 (ANS, 1993), que estabelece o valor mínimo de 12,5 MPa, para MOR. Para os painéis produzidos com partículas de vassoura e eucalipto, observou-se que, aumentando a porcentagem de vassoura, houve aumento do módulo de ruptura até a adição de $75 \%$. Os resultados da análise de variância indicaram que houve efeito significativo da composição dos painéis para o módulo de elasticidade (MOE), como se pode observar na Tabela 4. Ocorreu, também, efeito significativo da interação entre a composição dos painéis e o teor de adesivo para os produzidos com partículas de vassoura e eucalipto. Observa-se, na Tabela 5, que o teor de adesivo teve efeito significativo sobre o MOE. Observou-se que os menores valores médios encontrados para o MOE foram para os painéis produzidos com 100\% de partículas de vassoura, isto pode ser atribuído ao enovelamento das partículas de vassoura na etapa de formação do colchão, dificultando sua homogeneidade, contribuindo para a formação de espaços vazios, pela má distribuição das partículas dentro do colchão. Independentemente da composição das partículas, todos os painéis (Tabela 4), apresentaram valores médios acima do mínimo exigido pela norma ANSI/A 208.1/93 (ANS, 1993), que estabelece valores maiores que $22,70 \mathrm{MPa}$, para dureza Janka. Verificou-se para a composição vassoura e eucalipto, que à medida que se aumentou a porcentagem de partículas de vassoura, aumentou a dureza dos painéis, exceto para aqueles produzidos apenas com partículas de vassoura. Esse fato pode ser atribuído à maior área superficial da partícula de vassoura, contribuindo para melhor resistência superficial do painel quando submetido ao teste de dureza Janka. Na Figura 2 apresentam-se os valores médios do módulo de elasticidade em função da composição das partículas e do teor de adesivo. Observa-se que os painéis produzidos com $25 \%$ de vassoura / $75 \%$ de eucalipto e $100 \%$ de vassoura, apresentaram o maior e menor valor para MOE, respectivamente, quando adicionado $8 \%$ de adesivo às partículas. Isso ocorreu, provavelmente, em razão da geometria das partículas de vassoura e eucalipto e à desuniformidade na etapa de formação do colchão.

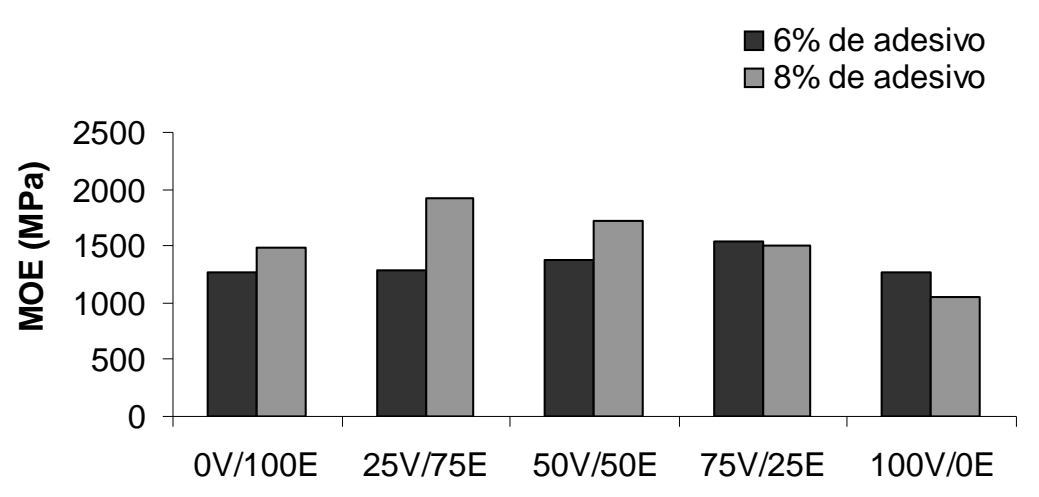

Composição(\%): Vassoura(V) x Eucalipto(E)

Figura 2 - Valores médios do Módulo de Elasticidade (MOE) em função da composição das partículas (vassoura x eucalipto) utilizando $6 \%$ e $8 \%$ de adesivo.

Figure 2 - Modulus of Elasticity (MOE) mean values as affected by particle composition (Eucalyptus and vassoura) using $6 \%$ and $8 \%$ of adhesive. 


\section{CONCLUSÕES}

Concluiu-se que o incremento de partículas de vassoura nos painéis teve efeito diferenciado em função das espécies utilizadas. A diferença entre a geometria das partículas de vassoura, eucalipto e paricá, influenciaram nas propriedades físicas e mecânicas dos painéis. $\mathrm{O}$ incremento da porcentagem de partículas de vassoura aos painéis produzidos com eucalipto não afetou a resistência à tração perpendicular, arrancamento de parafuso e dureza Janka. O incremento da porcentagem de partículas de vassoura aos painéis produzidos com paricá não afetou a resistência à dureza Janka e ao arrancamento de parafuso. Os resultados experimentais mostraram que o aumento no teor de adesivo na produção dos painéis de aglomerado contribuiu para melhorar algumas propriedades físicas e mecânicas. Apesar da dificuldade de formação do painel, em razão do enovelamento das partículas de vassoura, concluiu-se que o gênero Sida apresentou potencial para a produção dos painéis de aglomerado.

\section{AGRADECIMENTOS}

À CAPES pela concessão da bolsa de Mestrado. Aos funcionários dos laboratórios de Painéis e Energia da Madeira e Propriedades da Madeira da Universidade Federal de Viçosa.

\section{REFERÊNCIAS}

AMERICAN NATIONAL STANDARD. Mat-formed wood particleboard: specification ANSI/A 208.1. Gaithersburg: National Particleboard Association, 1993. 9 p.

\section{ASSOCIAÇÃO BRASILEIRA DE NORMAS TÉCNICAS.} NBR 14810-3: chapas de madeira aglomerada: métodos de ensaio. Rio de Janeiro, 2002.

BACCHI, O.; LEITÃO FILHO, H. F.; ARANHA, C. Plantas invasoras de culturas. Campinas: Círculo do Livro, 1982. v. $1,291 \mathrm{p}$.

BIANCHE, J. J. Propriedades de aglomerado fabricado com partículas de eucalipto (Eucalyptus urophylla), paricá (Schizolobium amazonicum) e vassoura (Sida spp.). 2009. 81 p. Dissertação (Mestrado em Ciência Florestal) - Universidade Federal de Viçosa, Viçosa, 2009.

CALDERAN, C. Vassoura (Sida sp). Disponível em: <http:// www.jardineiro.net/br/banco/sida_sp.php>. Acesso em: $8 \mathrm{dez}$. 2007.

Cerne, Lavras, v. 18, n. 4, p. 623-630, out./dez. 2012
COLI, A. Caracterização da madeira de paricá (Schizolobium amazonicum Huber ex. Ducke) e propriedades de chapas de partículas aglomeradas com diferentes proporções de fibra de coco (Cocus nucifera L.). 2007. 50 p. Dissertação (Mestrado em Ciência Florestal) Universidade Federal de Viçosa, Viçosa, 2007.

IWAKIRI, S. et al. Mistura de espécies na produção de chapas de partículas estruturais "waferboard". Revista do Setor de Ciências Agrárias, Curitiba, v. 14, n. 1/2, p. 107-114, 1995.

IWAKIRI, S. et al. Utilização de diferentes espécies do gênero pinus para produção de painéis "waferboard". IPEF, Piracicaba, n. 43/44, p. 65-69, jan./dez. 1990.

KELLY, M. W. Critical literature review of relationships between processing parameters and physical properties of particleboard. Madison: USDA, 1977. 66 p.

LORENZI, H. Plantas daninhas do Brasil: terrestres, aquáticas, parasitas, tóxicas e medicinais. 5. ed. Nova Odessa: Plantarum, 2000. 478 p.

NAUMANN, R. B.; VITAL, B. R.; CARNEIRO, A. de. C. O.; DELlA LUCIA, R. M.; SILVA, J. de C.; CARVALHO, A. M. M. L.; COLLI, A. Propriedades de chapas fabricadas com partículas de madeira de Eucalyptus urophylla S. T. Blake e de Schizolobium amazonicum Herb. Revista Árvore, Viçosa, v. 32, n. 6, p. 1143-1150, 2008.

SANTANA, M. A. E.; PASTORE, J. F. Adesivo de taninoformaldeído para aglomerados. Brasília: Instituto Brasileiro de Desenvolvimento Florestal, 1981. (Série Técnica, 2).

SANTOS, R. C. dos. Aproveitamento de resíduos da madeira de candeia (Eremanthus erythropappus) para produção de chapas de partículas. 2008. 159 p. Dissertação (Mestrado em Ciência e Tecnologia da Madeira) - Universidade Federal de Lavras, Lavras, 2008.

VITAL, B. R. Effects of species and panel densities on properties of hardwood particleboard. 1973. $111 \mathrm{f}$. Dissertation (Master of Science Forestry) - University of Wisconsin, Madison, 1973.

VITAL, B. R. Métodos de determinação da densidade da madeira. Viçosa, MG: UFV, 1984. (Boletim Técnico, 1).

VITAL, B. R.; HASELEIN, C. R.; DELLA LUCIA, R. M.

Efeito da geometria das partículas nas propriedades das chapas de madeira aglomerada de Eucalyptus grandis (Hill ex-Maiden). Revista Árvore, Viçosa, v. 16, n. 1, p. 88-96, 1992. 